\title{
Aroidene Maximillanae.
}

Unter obigem Titel wurde soeben ein Prachtwerk, sub auspiciis S. M. des Kaisers erscheinend, der Oeffentlichkeit übegeben ${ }^{1}$ ).

Als S. kais. Hoheit der Erzherzog Maximilian nach beendetem Krieg im Jahre 1859 sich zu einem Besuche Brasiliens entschloss, wollte er die Reise auch der Wissenschaft und den schönen Künsten nulzhar machen. Maler Selleny wurde eingeladen, den Erzherzog zu begleiten; eingeschifft als Bordarzt auf S. M. Dampfer "Elisabelh," welcher zur Expedition designirt war, erhielt ich den Auftrag, zugleich für die Botanik thätig zu sein, und Hofgärtner Maly wurde berufen, um beim Sammelgeschäft mitzuhelfen und namentlich die Acquisition lebender Gewächse für die k. k. Gärten von Schönbrunn zu besorgen. - Die Details dieser Reise findet man in den glanzvollen, leider unvollendeten Schilderungen derselben, welche aus der Feder Sr. k. Hoheit selbst stammen, ferner einige skizzenhafte Entwürfe, vorwiegend botanischer Objecte, in meiner Arbeit über die botanischen Ergebnisse der Expedition. Hier genüge nur die Bemerkung, dass sich dis botanische Ausbeutung auf die Umgebung von Rio (Petropolis) und Ilheos concentrirte, und dass namentlich die Riesenurwälder des letzteren Gebietes sich als eine reiche Fundstätte botanischer Schätze erwiesen haben.

Gleich nach vollendeter Reise wurde an die Arbeit gegangen. Mir ward die Beschreibung des botanischen Materials zugewiesen, - mit Ausnahme der Aroideen, deren Bearbeitung der berühmte Kenner dieser Pflanzenclasse, Director Schott, selbst übernommen hat. Ich konnte meine Arbeit bis zum Jahre 1866 fertig bringen ${ }^{2}$ ); aber die Aroideen traf ein eigenes Verhängniss. Während unserer Stationirung in Mexico, wohin ich Se. $k$. Hoheit den Erzherzog Ferdinand IIaximilian, nunmehr Kaiser Maximilian I. als Bordarzt S. M. Fregatte "Novara" begleitet, starb Schott.

Nach Wien im Herbst 1865 zur Vollendung meiner Arbeit zurückgekehrt, erhielt ich von Sr. M. dem Kaiser Max die schriftliche Ermächtigung zur Publication der Schott'schen Arbeit, und übernahm sofort Manuscript und Tafeln aus dem Schott'schen Nachlass, aber die mittlerweile eingetretenen kriegerischen Verhältnisse liessen mir kaum Zeit meine eigene Aufgabe zu vollenden, ich musste die Aroideen an Kotschy abtreten; auch Kotschy wurde bald vom Tode ereilt, die Aroideen gingen an Reissek über. Nach meiner Rückkunft von der ostasiatischen Expedition fand ich die Aroideen in den Händen Fenzl's, Reissek lag todtkrank darnieder und starb bald darnach; die Arbeit hatle nun volle Aussicht bald zum Ab-

1) Aufgelegt bei C. Gerold's Sohn in Wien.

2) Botanische Ergebnisse der Reise S. M. des Kaisers Maximilian I. nach Brasilien 1859-1860. - Die darin aufgenommene Beschreibung der Lichenen stammt von Kremplhuber, jene der Orchideen von Reichenbach fil. 
schluss gebracht und publicirt zu werden. Aber auch Fenzl erlahmte bald durch Alter und Krankheit, und musste - obwohl mit Widerstreben - die Arbeit an meinen Freund Peyritsch überlassen, den ich schon lange vorher dafür in Vorschlag gebracht, und welchem auch über Anempfehlung des Herrn Obersten Marinearztes Dr. Jilek von S. M. Cabinetskanzlei der Auftrag zur Vollendung des Werkes ertheilt wurde; und Peyritsch hat die Aufgabe in verhältnissmässig kurzer Zeit und gründlich gelöst.

Fenzl selbst erlebte das Erscheinen der Aroideen nicht mehr, er war der vierte in der Reihe der Botaniker - und wahrlich sie zählten zu den besten Männern Oesterreichs, welche über den ominösen Aroideen ins Grab sanken. Selleny, gleichfalls an dem Werke betheiligt, wurde dahingerafft, und den Schöpfer des Werkes, den edlen, mit Herzens- und Geistesgaben reich ausgestatteten Sprossen des österreichischen Kaiserhauses, erreichte vor Queretaro sein furchtbares Geschick. In dem vorliegenden Prachtwerk bewahrt dem kunstsinnigen Fürsten und den dahingeschiedenen an dem Bau betheiligten Meistern die Wissenschaft ein Denkmal, welches geweiht durch die Auspicien des Kaisers, ihren Ruhm der Nachwelt verkünden und zugleich Zeugniss ablegen wird von der Munificenz österreichischer Prinzen und von der Leistungskraft österreichischer Forscher.

Ueber das Werk selbst, welches mit kaiserlicher Munificenz ausgestattet - von dem grossen Kenner der Aroideen verfasst und in den Aleliers zweier weitberühmten Künstlerfirmen entstanden ist - was bleibt hier der Kritik viel zu sagen! sie muss sich auf einige historische Bemerkungen beschränken.

Die Aroideenausbeute bestand aus getrockneten Pflanzen, und in einer grossen Zahl lebender Gewächse, deren Knollen oder Samen nach Schönbrunn gebracht und hier ausgesäet und gepflegt wurden. Das Herbarium zählte nicht weniger als 84 Nummern (Arten), denn Maly hatte für seinen Herrn und Director besonders nach Aroideen gefahndet; die Mühe solche Massen dieser saftreichen und sonst sehr widerspanstigen Gewächse trocken zu bringen, war keine geringe, und sie war umsonst, denn Schott liess das Herbar ganz unberücksichligt und bielt sich bei seiner Arbeit nur an die lebenden Pflanzen; merkwürdiger Weise war dieses Herbar im Scholl'schen Nachlass nicht mehr aufzufinden. Für die auf der Reise Sr. kais. Hoheit acquirirten Aroideen wurde in Schönbrunn ein eigenes Gewächshaus hergerichtet, die Arten in der Reihenfolge ihres Aufblühens beschrieben, und von dem damals bei Sch ot t ständig beschäftigten Pflanzenzeichner Lieboldt porträtirt; hier sei noeh bemerkt, dass bis zum Jahre 1865 eine Menge der mitgebrachten Pflanzen noch nicht zur Blüthe gelangt war, dass also über diese Zeit hinaus ein Abschluss des Werkes durch Schott, falls er am Leben geblieben wäre, noch lange nicht zu gewärtigen stand.

Das Schott'sche Manuscript bestand in losen Blättern, je eines für jede Art; Diagnose und Beschreibungen waren mit schöner deut- 
licher Handschrift copirt, und jede Species mit jener Gründlichkeit und Ausführlichkeit behandelt, welcho den Altmeister der Aroideen liennzeichnete. Bei dem grösseren Theil der Arten war die Beschreibung vollständig, bei manchen nur fragmentarisch, oder es fand sich nur die Diagnose vor, in seltenen Fällen fehlte auch diese.

Die Abbildungen - oft in mehreren Blätlern zerstreut - lagen alle vollständig vor, und nach diesen Abbildungen und ferner durch Zuhilfenahme anderweitiger Quellen (namenllich Scholt's Prodr., Gen., Syn. Aroid. und die im vorigen Jahre erschienene Aroid. - Flora Brasil. von Engler) mussten di: fehlenden oder fragmentarischen Beschreibungen ersetzt und completirt werden. Mit Ausdauer und Geschick hat diese Aufgabe Prof. Peyritsch gelöst; von ihm rühren auch die Zusammenstellung der Literatur und die Bezeichnung weiterer Fundorte her; die Eriklärung der Abbildungen hat noch Reissek besorgt.

In den Druck der Tafeln theilten sich die Firmen Hartinger und Reiffenstein; es galt ein vaterlandisches Kunstwerk in seiner Vollendung herzustellen, vielleicht auch eine kleine Aemulation, welche der Sache nur zum besten dienen konnte, liess sie alle Kraft anspannen, und so liam es, dass aus den Tafeln eben so viele Meisterwerke wurden, welche der österreichischen Chromolithographie alle Ehre machen. Dass aber auch die botanische Exactheit in den Darstellungen besonders der Analysen gewahrt wurde, dafür bürgt die bekannte Genauigkeit und Scrupulositat Reissek's, der den Druck überwachte, und ich kann mir lehhaft vorstellen, dass er unseren beiden Lithographen gar viel zu schaffen inachte. Besonders gefällig sind jene Bilder, welche die Pflanze für sich allein darstellen, während die Analysen auf einer zweiten Tafel Platz fanden. Wo auf Einem Blatt beide vereinigt sind, ersiheint das Habitusbild durch die gleichfalls farbigen Analysen etwas gedrückt.

Das Titelbild, von Selleny gezeichnel, bringt eine Partie des Urwaldes in seiner vollen Prachtentwicklung zur Anschiaung. Die übrigen 42 Tafeln enthalten 30 Arten; darunter sind nicht weniger als 18 Arten neu beschrieben, einige der älteren Species (z. B. die Staurosligmaarten) erhielten durch die Aufstellung neuer Galtungen auch neue Namen.

Zum Schluss noch ein Wort zur Abwehr eines Vorwurfs, den man gegen das Werli vielleicht erheben könnte, nämlich, dass in Folge der durch eine Verkettung funester Umstände herbeigeführten Verspitung seiner Publication dasselbe an wissenschaftlichem Werth eingebüsst hatte; dieser Einwurf ist grundlos; indem Schott die neuen Arten sofort in der "Oesterr. botan. Zeitschrift" und in der "Bonplandia" veröffentlicht, und damit sich selbst die Priorităt und seinem posthumen Werk die wissenschaftliche Bedeutung sicher gestellt hat.

Dr. Waw ra. 